\title{
Comparison of adequacy of birth weight for gestational age according to different intrauterine growth curves
}

Micaely Cristina dos Santos Tenório 1

iD https://orcid.org/0000-0002-1771-489X

Carolina Santos Mello 2

https://orcid.org/0000-0001-6461-1749

Juliana Célia de Farias Santos 3

https://orcid.org/0000-0003-3679-0158

Alane Cabral Menezes de Oliveira 4

https://orcid.org/0000-0002-7497-919X

1,3,4 Faculdade de Nutrição. Universidade Federal de Alagoas. Campus A. C. Simões, BR 104 Norte, Km 96,7, Tabuleiro dos Martins. Maceió, Alagoas, Brasil. CEP: 57.072-
900. E-mail: alanecabral@gmail.com

2 Universidade Federal da Bahia. Escola de Nutrição. Salvador, Bahia, Brasil.

\begin{abstract}
Objectives: to compare the assessment of the adequacy of birth weight for gestational age according to different intrauterine growth curves.

Methods: across-sectional study, which analyzed gestational and neonatal information from 344 mother-newborn binomials. Birth weight data were analyzed using the International Fetal and New Born Growth Consortium for the 21st Century (INTERGROWTH-21st) and compared with the growth curves proposed by Alexander et al. and Fenton \& Kim. Newborns were classified as small for gestational age (SGA), suitablefor gestational age (SUGA) or large for gestational age (LGA).

Results: among the newborns, $51.2 \%$ were male, and $93.0 \%$ were born at term. Higher prevalence of SUGA and LGA and lower SGA was found by the INTERGROWTH-21st curves when compared to the references of Fenton \& Kim and Alexander et al. Moderate agreement was observed in detecting birth weight by different growth curves.

Conclusions: there was a lower detection of SGA infants and a higher screening, especially of LGA infants, in the INTERGROWTH-21st evaluation, when compared to the growth curves of Fenton \& Kim and Alexander et al.
\end{abstract}

Key words Growth charts, Gestational age, Newborn

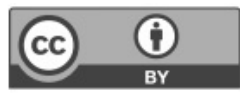




\section{Introduction}

Birth weight is one of the health indicators that most influences the health-disease process in the first years of life. ${ }^{1}$ Its extremes are associated with higher rates of neonatal and postnatal morbidity and mortality, in addition to childhood and adult age morbidity. ${ }^{2}$ Additionally, birth weight has been evaluated as a predictor of the development of chronic noncommunicable diseases in adolescents and adults. ${ }^{3}$

For the birth weight classification of newborns of different gestational ages, the use of intrauterine and/or neonatal growth curves is recommended. However, these are usually based on specific population data,4-8 which makes it difficult to compare across different populations.Among the various curves, we highlight the Fenton \& Kim curves, 4 which were developed from a meta-analysis with a representative sample of newborns from studies conducted in six countries, being the most frequently used in Brazil in recent years; and the Alexander et al. 5 curves, constructed using data from a significant number of live births of single pregnancies of American women from different ethnic groups, using the last menstrual date (LMD) as the method for defining gestational age.

Recently, newcurves, named International Fetal and New Born Growth Consortium for the 21 st Century (INTERGROWTH-21st) were published, 9 allowing anthropometric assessment in the fetal, neonatal and postnatal period of children, regardless of gestational age at birth. These, as well as the curves recommended by the World Health Organization (WHO), in Multicentre Growth Reference Study, 10 were built from data from different countries and ethnic groups, including Brazil, and are characterized as a referral where different populations can be evaluated and compared. However, despite its relevance, there is still no recommendation on the growth curve to be used to assess newborns in Brazil. In this context, this article aims to compare the assessment of birth weight adequacy for gestational age according to different curves.

\section{Methods}

A cross-sectional study conducted with newborns of pregnant women attended at the public health network in the city of Maceio, State of Alagoas, in 2014. The study is part of a larger research from Brazilian National Health System (Sistema Único de Saúde - SUS) entitled "Nutritional status, weight gain and eating behaviour of pregnant women from Maceió-Alagoas: impact on the health of the motherchild binomial" (Edital PPSUS number 60030 000741/2013).

In 2014 the municipality was strategically organized into eight health districts, with a total of 60 Basic Health Units (BHU). For the sample selection of the studied population, a random draw of $50 \%$ of the total BHU, by sanitary district, was performed. Once the selected units were defined, a score was established according to the number of pregnant women registered in each unit, according to the list provided by the Municipal Secretary of Health, so that those units with more registered pregnant women contributed, proportionally, with a higher number in the sample. The recruitment and data collection of pregnant women were performed through non-random interviews on the days established for prenatal consultations at the BHU, and the pregnant women were invited for voluntary participation in the study. Newborn data were subsequently collected from the Municipal Health Department's Registration System.

As inclusion criteria, the study included newborns of pregnant women attended at the municipal public health network of Maceio, coming from a single pregnancy, and newborns with congenital diseases or neonatal complications were not included. Pregnant women with neurological problems that impededthe interview and /or who presented physical limitations for anthropometric evaluation were excluded.

The sample size calculation was performed a posteriori with the aid of the Epi Info version 7.0 program, considering the prevalence of small newborns for gestational age (SGA) of $12.0 \%, 11 \mathrm{a}$ sampling error of $3 \%$ and an interval of $95 \%$ confidence, being necessary 331 newborns.

The data obtained from the newborns were gestational age at birth, modeof delivery and birth weight. Birth weight was classified according to the growth curves proposed by INTERGROWTH-21st, 9 Fenton \& $\mathrm{Kim}^{4}$ and Alexander et al., 5 and newborns with birth weight below the $10^{\text {th }}$ percentile were characterized as small for gestational age (SGA); suitable for gestational age (SUGA) those between the $10^{\text {th }}$ and $90^{\text {th }}$ percentiles; and large for gestational age (LGA) newborns above the 90th percentile. In this study, we considered as a reference growth curve the INTERGROWTH-21 st (2014), ${ }^{9}$ because it presents a representative sample of the Brazilian population in its construction.

For the classification of gestational age at birth, those with a gestational age inferior than 37 weeks 
were considered preterm, term those with a gestational age between 37 and 41 weeks, and post-term those with a gestational age of 42 weeks or more. 12

Statistical analyzes were performed with the aid of the STATA ${ }^{\circledR}$ version 13.0, adopting a confidence level of $95 \%(\alpha=0.05)$. The degree of agreement between the methods (growth curves) expressed by the weighted Kappa $(\mathrm{K})$ value was measured considering the following cut off points: 0-0.39 poor agreement; 0.40-0.59 moderate agreement; 0.600.79 substantial agreement and 0.80-1.00 near perfect agreement. 13

The project was approved by the Research Ethics Committee of the Federal University of Alagoas (CAAE Number 18807.113.3.0000.5013).

\section{Results}

A total of 344 newborn were evaluated, with 176 male children $(51.2 \%)$, with mean birth weight and length of $3240 \mathrm{~g}( \pm 550 \mathrm{~g})$ and $48.67 \mathrm{~cm}( \pm 3.32 \mathrm{~cm})$, respectively. There was a higher frequency of term births $(n=320 ; 93.0 \%)$, with a median of 39 (minimum of 34 and maximum of 43 ) gestational weeks at birth. Twenty (5.8\%) children were born preterm and four (1.2\%) postpartum.

Table 1 shows the comparisons of birth weight classifications between the different curves. When the INTERGROWTH-21st 9 and Fenton \& Kim 4 ratings were compared, $4.9 \%$ vs $16.9 \%$ of the concepts were SGA $(p<0.001) ; 85.2 \%$ vs $73.5 \%$ were AGA $(p<0.001)$ and $9.9 \%$ vs $9.6 \%$ were LGA $(p<0.001)$. On the other hand, when compared to INTERGROWTH-21st 9 and Alexander et al.,5 it was found, respectively, that $4.9 \%$ vs $18.6 \%$ of the concepts were SGA $(p<0.001) ; 85.2 \%$ vs $74.1 \%$ were SUGA $(p<0.001)$ and $9.9 \%$ vs $7.3 \%$ were LGA $(p<0.001)$.

Table 2 shows the agreement values between birth weight classifications according to the different curves. As can be seen, there was a moderate agreement between the INTERGROWTH-21st 9 and Fenton $\& \mathrm{Kim}^{4}$ curves $(\mathrm{K}=0.5625 ; p<0.001)$ and between INTERGROWTH-2 1 st 9 and Alexander $e t$ $a l .5(\mathrm{~K}=0.5581 ; p<0.001)$.

\section{Discussion}

In assessing birth weight of newborns, by different growth curves, higher prevalence of SUGA and LGA and lower SGA was found by INTERGROWTH$21^{\text {st } 9}$ when compared to the references of Fenton \& $\mathrm{Kim}^{4}$ and Alexander et al. 5 Moderate detection of birth weight between INTERGROWTH-2 1 st 9 and Fenton \& Kim, 4 and between INTERGROWTH$21^{\mathrm{st}} 9$ and Alexander et al. 5

Some decades ago, birth weight according to pre-established cut-offs have been used in clinical practice. 14 More recently, the publication of new INTERGROWTH-21st 9 curves has made it possible to assess neonatal growth. It is important to emphasize that these criteria consider, in addition to anthropometric data obtained at birth and gestational age at birth, the gender of the newborn, which constitutes a limitation in the use of the Fenton \& $\mathrm{Kim}^{4}$ and Alexander et al. 5

Regarding the curves for birth weight classification used in this research, it is noteworthy that, in the assessment of fetal weight estimation, the INTERGROWTH-21 st9 curve was developed from multicentric data of pregnancies with minimum criteria regarding age, height, weight, diet and pre-existing clinical conditions, being excluded any complications that could interfere with fetal size. Fenton \& $\mathrm{Kim}^{4}$ curves were developed from a meta-analysis, including studies from six developed countries, which allows this method to be valid for external purposes. In contrast, the curve of Alexander et al. 5

Table 1

Comparison between the birth weight categories of newborns treated in the public health network according to the curves of INTERGROWTH-21st 9, Fenton \& Kim4 and Alexander et al.5 Maceio, Alagoas, Brazil, 2014.

\begin{tabular}{|c|c|c|c|c|c|c|c|c|c|c|}
\hline \multirow[t]{2}{*}{ Weight categories } & \multicolumn{2}{|c|}{ INTERGROWTH-21st 9} & \multicolumn{2}{|c|}{ Fenton $\& \mathrm{Kim}^{4}$} & \multicolumn{2}{|c|}{ Alexander et al. 5} & \multirow[t]{2}{*}{$\chi^{2 *}$} & \multirow[t]{2}{*}{$p^{*}$} & \multirow[t]{2}{*}{$\chi^{2 * *}$} & \multirow[t]{2}{*}{$p^{* *}$} \\
\hline & $\mathrm{n}$ & $\%$ & $\mathrm{n}$ & $\%$ & $\mathrm{n}$ & $\%$ & & & & \\
\hline SGA & 17 & 4.9 & 58 & 16.9 & 64 & 18.6 & 88.18 & $<0.001$ & 78.24 & $<0.001$ \\
\hline SUGA & 293 & 85.2 & 253 & 73.5 & 255 & 74.1 & 96.16 & $<0.001$ & 86.24 & $<0.001$ \\
\hline LGA & 34 & 9.9 & 33 & 9.6 & 25 & 7.3 & 177.83 & $<0.001$ & 204.10 & $<0.001$ \\
\hline
\end{tabular}

Chi-square test $\left(\chi^{2}\right), p<0.05$ as significant. SGA $=$ Small for Gestational Age, SUGA = Suitable for Gestational Age, LGA $=$ Large for Gestational Age.

*Comparison between INTERGROWTH-21st9 and Fenton \& Kim. 4

** Comparison between INTERGROWTH-21st9 and Alexander et al.5 
Agreement between the birth weight categories of newborns treated in the public health network according to the curves of INTERGROWTH-21st9, Fenton \& Kim ${ }^{4}$ and Alexander et al.5 Maceio, Alagoas, Brazil, 2014.

\begin{tabular}{lccc}
\hline Variables & $\mathbf{K}^{*}$ & Agreement (\%) & $\boldsymbol{p}^{*}$ \\
\hline INTERGROWTH-21st9/ Fenton \& Kim4 & & & \\
\hline INTERGROWTH-21st9/ Alexander et al.5 & 0.5625 & 92.01 & $<0.001$ \\
\hline
\end{tabular}

* Weighted Kappa agreement test (K), $p<0.05$ as significant.

was constructed from a United States (USA) database, showing that the use of this curve in specific ethnic groups would not be adequate.

When comparing the two criteria 4,5 with the new proposals of INTERGROWTH-21st9, in the assessment of birth weight, an average prevalence of 3.6 times higher SGA and 1.2 times lower LGA was observed when the criteria Fenton \& $\mathrm{Kim}^{4}$ and Alexander et al. 5 Similarly, a study by Kozuki et al., 15 which evaluated birth weight data from children participating in 16 cohorts by INTERGROWTH-21st 9 and two other North American growth curves, found a pooled prevalence of SGA infants in $23.7 \%$ of the children, when using the INTERGROWTH-21st9 curves and, on average, $34.4 \%$, when the other American curves were used. Thus, the authors verified a reduction of about $30 \%$ in the prevalence of SGA among the studied cohorts, when the new referential was used.

These findings reinforce the observation that the new INTERGROWTH-21 st 9 curves have a larger shift to the right side to be more sensitive in screening for LGA newborns and, consequently, reducing the diagnosis of SGA. A similar observation was discussed by other authors when comparing the growth curves of the WHO Multicentre Growth Reference Study 10 with the old National Center for Health Statistics (NCHS) 16 and Centers for Disease Control and Prevention (CDC) ${ }^{17}$ standards, where it was shown that WHO curves present greater sensitivity in detecting overweight when compared to other references. ${ }^{18,19}$ These findings are presented within the context of the nutritional transition observed in recent decades, from the perspective of the need to prevent and address the obesity epidemic. 20

It has been well described in the literature that complications in intrauterine life are closely related to human development, from childhood to adulthood. ${ }^{1}$ However, to observe this phenomenon, known as fetal programming, further clarification of the factors associated with different pathological outcomes is still necessary. These factors can be attributed to conditions inherent to the mother and her inadequate nutritional status, as well as the occurrence of endocrine disorders, such as gestational diabetes, can be listed, which may lead to an excessive or limited supply of nutrients to the fetus, impairing its normal evolution. In addition to this, other complications in pregnancy associated with the placental condition, such as intrauterine growth restriction, preeclampsia and hypoxia, are important challenges in the care of the maternal-fetal binomial.21

Considering strong evidences that point out the importance of the first 1000 days of life in the performance of interventions capable of preventing childhood morbidity and mortality and health problems throughout life, which are defined as a window of opportunity, ${ }^{22}$ the new INTERGROWTH-21 st 9 curves become favorable in early detection of overweight, allowing strategies to be applied to minimize its long-term effects, more precisely in the development of noncommunicable chronic diseases. Corroborating this understanding, Francis et al.23 found, based on data from 10 countries, higher LGA rates with the use of the INTERGROWTH-21st9 reference when compared to the use of the custom standard English curve (GROW).

On the other hand, the birth of SGA, associated with unhealthy living conditions, low number of prenatal consultations and low maternal education is still a serious public health problem, being a reality in the Northeast region of Brazil, 24 which raises the hypothesis that the use of more sensitive criteria for detection could be more accurate. In this context, the Fenton \& Kim, ${ }^{4}$ Alexander et al. ${ }^{5}$ curves that track more SGA could be more indicated in the detection of newborns as eligible for greater health and nutrition-related care.

The unified use of the same growth curves for the assessment of the nutritional status of children at birth is controversial, when it is a reference where distinct populations can be evaluated and compared. The optimal fetal growth and development exists when intrauterine conditions, inherent to maternal 
health and nutrition conditions, are adequate. In this case, it would be justifiable and appropriate to use the same framework in different populations.25 However, in countries with differing levels of social and economic development, this seems to be a problem.

Another aspect that needs to be analyzed is about the practical use of these tools. The classification criteria used in the present study for neonatal anthropometric assessment are discordant in the assessment of birth weight. In this study, we found that the curves of Fenton \& $\mathrm{Kim}^{4}$ and Alexander et al. would be better applied in SGA screening and, on the other hand, INTERGROWTH-21st 9 would be important for LGA screening. In this context, it is important to highlight that usually in health services in Brazil, it is commonly verified the assessment based only on the birth weight of the child, classifying as low birth weight those weighing less than $2500 \mathrm{~g}$, and macrosomic children born with weight equal to or greater than $4000 \mathrm{~g}, 25$ which is also a method of limitations because it does not reflect all aspects of fetal growth and development. 1 The results of the present study do not allow a careful evaluation about which method would be more appropriate to characterize the nutritional status of the Brazilian newborn and this aspect may be the object of future studies.

\section{References}

1. Organización Mundial de la Salud. Reunión consultiva técnica de la OMS sobre la elaboración de una estrategia de promoción del desarrollo fetal óptimo. (2003: Ginebra, Suiza). Promoción del desarrollo fetal óptimo: informe de una reunión consultiva técnica. OMS: Ginebra; 2006. 59p.

2. Melo ASO, Assunção PL, Gondim SSR, Carvalho DF, Amorim MMR, Benicio MHDA, Cardoso MAA. Estado nutricional materno, ganho de peso gestacional e peso ao nascer. Rev Bras Epidemiol. 2007; 10(2): 249-57.

3. Belbasis L, Savvidou MD, Kanu C, Evangelou E, Tzoulaki L. Birth weight in relation to health and disease in later life: an umbrella review of systematic reviews and metaanalyses. BMC Med. 2016; 14 (1): 147.

4. Fenton TR, Kim JH. A systematic review and meta-analysis to revise the Fenton growth chart for preterm infants. BMC Pediatr. 2013; 13: 59.

5. Alexander GR, Himes JH, Kaufman RB, Mor J, Kogan M. A United States national reference for fetal growth. Obst Gynecol. 1996; 87 (2): 163-8

6. Lubchenco LO, Hansman C, Dressler M, Boyd E. Intrauterine growth as estimated from liveborn birth-weight data at 24 to 42 weeks of gestation. Pediatrics. 1963; 32: 793-800.
Finally, newborn growth and optimal development should be routinely monitored with consistent tools to assist in the necessary neonatal care, as during this period growth as a continuous process may suffer interferences. To monitor growth, anthropometric standards are needed to assess adequacy levels and growth deviations, but, above all, that are compatible with the local reality and of each type of health service. Also, it should be noted that monitoring the growth curve of the child is more relevant than just the comparison with references.

It is important that studies with similar characteristics be carried out in other population groups, since different findings can be glimpsed, due to socioeconomic and cultural heterogeneities. Thus, it is intended to assist in the evaluation of the better referenceto be adopted in epidemiological studies in the country and in health services.

\section{Authors' contribution}

Tenório MCS - Data collection and article writing. Mello CS - Article writing and critical review. Santos JCF - Critical Review. Oliveira ACM - Study design, article writing and critical review. All authors approved the final version of the manuscript.

7. Usher R, McLean F. Intrauterine growth of live-born Caucasian infants at sea level: standards obtained from measurements in 7 dimensions of infants born between 25 and 44 weeks of gestation. J Pediatr. 1969; 74 (6): 901-10.

8. Williams RL. Intrauterine growth curves: intra- and international comparisons with different ethnic groups in California. Prev Med. 1975; 4 (2): 163-72.

9. Villar J, Ismail LC, Victora CG, Ohuma EO, Bertino E, Altman DG, et al. International standards for newborns weight, length, and head circumference by gestational age and sex: the Newborns Cross-Sectional Study of the INTERGROWTH-21st Project. Lancet. 2014; 384 (9946): 857-68.

10. WHO (World Health Organization), Multicentre Growth Reference Study Group. WHO Child growth standards based on length/height, weight and age. Acta Paediatr. 2006; 450 (Suppl): 76-85.

11. Tuzun F, Yucesoy E, Baysal B, Kumral A, Duman N, Ozkan H. Comparison of INTERGROWTH-21 and Fenton growth standards to assess size at birth and extrauterine growth in very preterm infants. J Matern Fetal Neonatal Med. 2017; 30 (1): 1-12.

12. WHO (World Health Organization). Public health aspects of low birth weight. Technical Report Series $n^{0} 217$. Geneve; 1961. 
13. Landis JR, Koch GG. The measurement of observer agreement for categorical data. Biometrics. 1977; 33 (1): 159-74

14. Nilson LG, Warmling D, Oliveira MSV, Gouveia GDA. Proporção de baixo peso ao nascer no Brasil e regiões brasileiras, segundo variáveis sócio-demográficas. Rev Saúde Públ Santa Cat. 2015; 8 (1): 69-82.

15. Kozuki N, Katz J, Christian P, Lee ACC, Liu L, Silveira MF, et al. Comparison of US Birth Weight References and the International Fetal and Newborn Growth Consortium for the 21st Century Standart. JAMA Pediatr. 2015; 169 (7): e151438.

16. Hamill PV, Drizd TA, Johnson CL, Reed RB, Roche A, Moore WM. Physical growth: National Center for Health Statistics percentiles. Am J Clin Nutr. 1979; 32 (3): 607-29.

17. Kuczmarski RJ, Ogden CL, Guo SS, Grummer Strawn LM, Flegal KM, Mei Z, Wei R, Curtin LR, Roche AF, Johnson CL. 2000 CDC growth charts United States: Methods and development. National Center for Health Statistics. Vital Health Stat. 2002; 246: 1-190.

18. Sperandio N, Santana LFR, Franceschini SCC, Priori SE. Comparação do estado nutricional infantil com utilização de diferentes curvas de crescimento. Rev Nutr. 2011; 24 (4): 565-74.

19. Oliveira GJ, Barbiero SM, Cesa CC, Pellanda LC. Comparação das curvas NCHS, CDC e OMS em crianças com risco cardiovascular. Rev Assoc Med Bras. 2013; 59 (4): $375-80$.
20. Adamo KB, Ferraro ZM, Goldfield G, Keely E, Stacey D, Hadjiyannakis S, Jean-Philippe S, Walker M, Barrowman NJ. The maternal obesity management (MOM) trial protocol: a lifestyle intervention during pregnancy to minimize downstream obesity. Contemp Clin Trials. 2013; 35:87-96.

21. Perrone S, Santacroce A, Picardi A, Buonocore G. Fetal programming and early identification of newborns at high risk of free radical-mediated diseases. World J Clin Pediatr. 2016; 5 (2): 172-81.

22. Pietrobelli A, Agosti M. Nutrition in the First 1000 Days: Ten Practices to Minimize Obesity Emerging from Published Science. Int J Environ Res Public Health. 2017; 14 (12): 1491.

23. Francis A, Hugh O, Gardosi J. Customized vs INTERGROWTH-21 st standards for the assessment of birthweight and stillbirth risk at term. Am J Obstet Gynecol. 2018, 218 (2 Suppl.): S692-S9.

24. Viana KJ, Taddei JAAC, Cocetti M, Warkentin S. Peso ao nascer de crianças brasileiras menores de dois anos. Cad Saúde Pública. 2013; 29 (2): 349-56.

25. WHO (World Health Organization). Physical status: the use and interpretation of anthropometry. Technical Reports Series, 854. Geneva; 1995.

Received on February 14, 2019

Final version presented on August 16, 2019

Approved on September 30, 2019 